\title{
Simonization for signal design
}

\author{
P S MOHARIR ${ }^{1}$, V M MARU $^{1}$ and R SINGH ${ }^{2}$ \\ ' National Geophysical Research Institute, Uppal Road, Hyderabad 500007, \\ India \\ ${ }^{2}$ School of Computer Science, Carnegie Mellon University, 5000, Forbes \\ Avenue, Pittsburgh, PA 15213, USA \\ e-mail: postmast@csngri.ren.nic.in; rsingh@cs.cmu.edu
}

MS received 13 November 1997; revised 12 May 1998

\begin{abstract}
Ternary sequences have been searched earlier with good merit factor or alternatively good discrimination as the search criterion. When one of these measures is a search criterion, the other measure takes generally a poor value. As both of them are quantifications of the abstract notion of a sequence with peaky autocorrelation, it is better to look for sequences with good values of merit factor and discrimination simultaneously, without optimizing either measure. This is called Simonization. Simonized ternary sequences are listed.
\end{abstract}

Keywords. Ternary sequences; signal design; Windows; Simonization.

\section{Introduction}

Optimization has been a powerful theme in science, because the latter consists of learning how effectively our models can be imposed on the external reality, and this analysis can be posed as an optimization problem. The problem has relevance also in the science of the artificial because the artifacts to be created or searched have to optimize the desideratum. But the problem of optimization is beset with two difficulties. First, what we can readily get is a local optimum and, whereas there are techniques of getting untrapped from a local optimum, they amount to providing a non-local and not really a global optimization. Second, there is no way of knowing what we should be optimizing. That there should be a good match between our model and the external universe is a qualitative desire and setting an objective function to be optimized is an attempt to quantify it. There is never any unique conversion of a qualitative desire of match to a quantitative measure of it. One believes that various quantitative desiderata should give only slightly different results, or alternatively that when one of them is optimized, the other should be only slightly suboptimal. In the context of the signal design problem it is shown that this belief is grossly misplaced.

For both these reasons, there is a school led by Simon (Simon 1956; Pearl 1985) subscribing to a view that one should not make a fetish of optimality and be content with 
satisficing. Whereas this is a good step, the word suggesting the change is somewhat artificial. Here the word would be replaced by an eponymic term Simonization, in honour of Simon, the propounder of the sciences of the artificial. It is possibly necessary to note that it has nothing to do with Simony, a term named after another Simon by Wiener (1964).

In the present context, signal design is a problem of getting sequences with peaky autocorrelation. The peakiness of the autocorrelation is definable by many criteria. Two of them are discrimination and merit factor. Optimization techniques have been applied recently to the classical signal design problem (Bernasconi 1987; De Groot et al 1992) in the context of binary sequences. Ternary sequences with large merit factors were obtained earlier by S-K-H algorithm (Moharir et al 1996; Moharir \& Subba Rao 1997). They were further improved by some untrapping techniques (Moharir et al 1997) to make the results of search nonlocal. It is easy in these algorithms to replace the objective function by discrimination, so that sequences with good discrimination are obtained. Not only that sequences having good merit factor and discrimination are different, in general, but that sequences having the best merit factor have very poor discrimination and vice versa. If both merit factor and discrimination are regarded as satisfactory measures of peaky autocorrelation, but actually disagree so severely, what is to be done? This paper makes a proposal in this context.

Ternary sequences with good merit factors have been listed earlier (Moharir et al 1996, 1997a; Moharir \& Subba Rao 1997). The length, the merit factor and the discrimination values for some of them are listed in table 1 . Merit factor values are very good, but the search was conducted for that purpose. The discrimination values did not figure in the search criterion, and are very poor.

Ternary sequences with large discrimination also have been listed earlier (Moharir et al 1997b), and the work has continued further. The length, the discrimination and the merit factor values for some of them are listed in table 2 . The values of the discrimination are

Table 1. Details of some of the ternary sequences obtained with an idea of getting good merit factor values.

Discrimination values did not figure in the search algorithm. They were merely calculated at the end.

\begin{tabular}{ccc}
\hline Length & Merit factor & Discrimination \\
\hline 275 & 13.1979 & 12.8824 \\
696 & 12.9600 & 9.9412 \\
699 & 12.5989 & 11.4091 \\
702 & 12.5603 & 12.5000 \\
277 & 12.2967 & 13.7500 \\
705 & 12.0051 & 13.7500 \\
698 & 11.7248 & 13.3784 \\
720 & 11.6311 & 8.8136 \\
768 & 11.3932 & 8.1875 \\
695 & 11.3740 & 10.8696 \\
1112 & 11.3302 & 12.8906 \\
299 & 10.9845 & 12.8889 \\
1120 & 10.9251 & 13.2903 \\
1089 & 10.7466 & 14.3409 \\
474 & 10.1154 & 16.4375 \\
\hline
\end{tabular}


Table 2. Details of some of the ternary sequences obtained with an idea of getting good discrimination values.

Merit factor values did not figure in the search algorithm.

They were merely calculated at the end.

\begin{tabular}{lcc}
\hline Length & Discrimination & Merit factor \\
\hline 3317 & 55.5429 & 2.9284 \\
3569 & 54.4464 & 2.9192 \\
3599 & 53.5357 & 3.1182 \\
3281 & 53.2632 & 2.6033 \\
3335 & 52.7632 & 2.6744 \\
3774 & 52.1967 & 2.6523 \\
3824 & 51.3387 & 2.4508 \\
4003 & 50.1940 & 2.1634 \\
3953 & 49.7910 & 2.2152 \\
2181 & 47.7333 & 4.0500 \\
1567 & 47.5455 & 4.6568 \\
1108 & 45.6471 & 4.9430 \\
1180 & 45.0000 & 5.3183 \\
1633 & 45.1538 & 3.5092 \\
1518 & 43.9200 & 3.6580 \\
\hline
\end{tabular}

good, as they constituted the search criterion, but the merit factor values, which were not part of the objective function, are very poor.

That is, if both discrimination and merit factor are regarded as acceptable quantitative measures of peakiness of the autocorrelation, they do not agree much. Further, as the length increases, it is more and more difficult to get large merit factors but easier to get larger and larger discriminations. So, does it become easier or more difficult to get peaky autocorrelations, as the length increases? The questions lie in the domain of multiobjective optimization, on which there is plenty of classical work (Zeleny 1974; Cohon 1978; Ching-Lai \& Masud 1979), before the advent of techniques of the so-called global optimization, and also application of some of these ideas in conjunction with these later techniques (Srinivas \& Deb 1995; Weile \& Michielson 1996). The work reported here could be regarded to have a similar motivation, in the area of signal design.

\section{Window on simonization}

When two objective functions, which are both quantitative measures of the same qualitative desire. differ widely, one way is not to trust either of them exclusively. What could be done is illustrated in this section with the synthetic problem of choice of a window. This problem is not entirely unrelated to radar, as amplitude-weighting, either at the transmitter or the receiver, or at both ends, is used in radar for better suppression of the sidelobes (Cook \& Bernfeld 1967). Window (Papoulis 1977) is a even function $w(t)$ defined on the temporal support $[-1.1]$, such that $w(0)=1, w(1)=0$ and in $[0,1]$ it is monotonically nonincreasing. It is easy to see that if $w_{1}(t)$ and $w_{2}(t)$ are two windows, then their convex combination

$$
w(t)=\alpha w_{1}(t)+(1-\alpha) w_{2}(t), 0 \leq t \leq 1,0 \leq \alpha \leq 1 .
$$



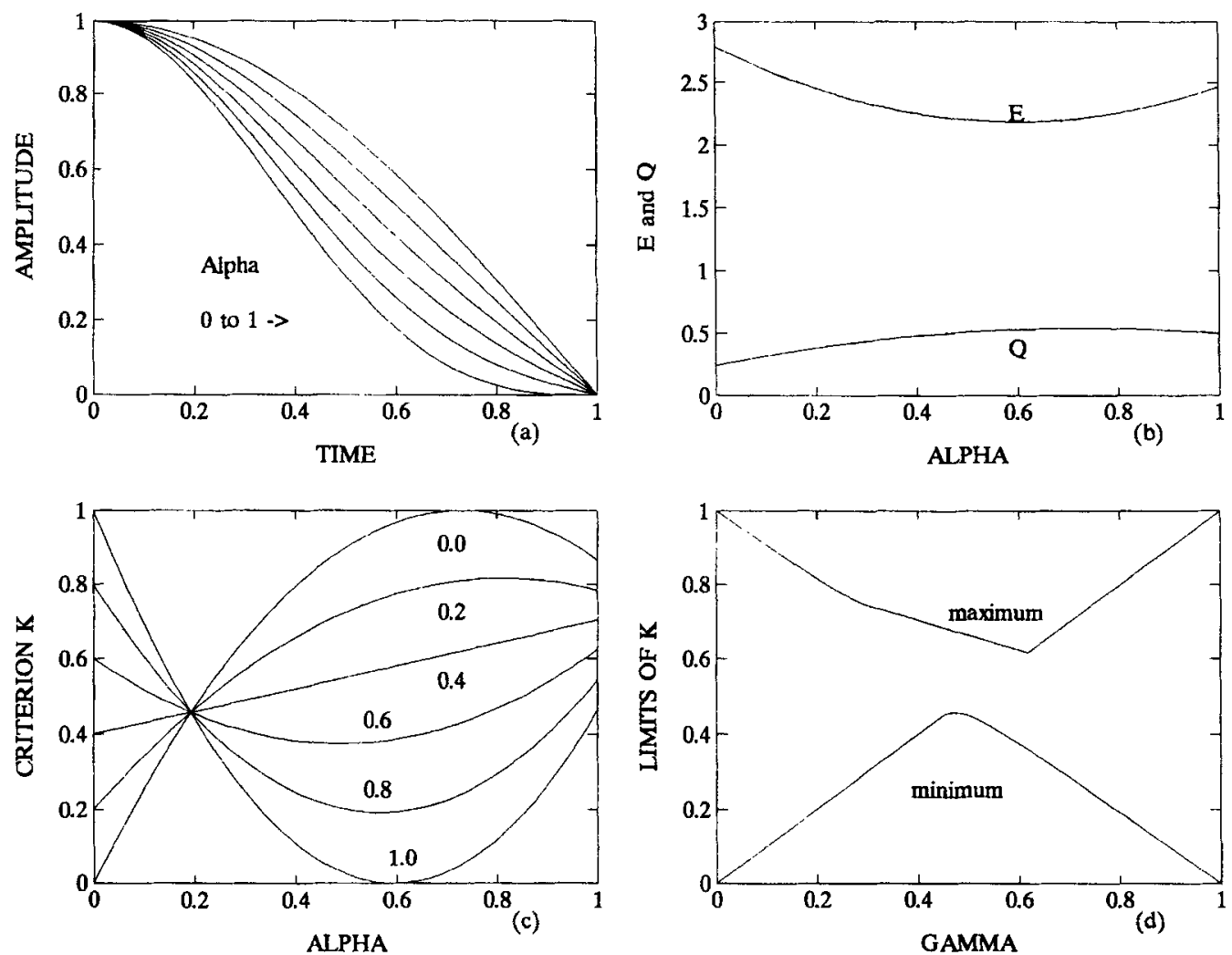

Figure 1. (a) Windows $w_{1}(t)$, and $w_{2}(t)$ and some of their convex combinations $w(t)$. (b) Variance compensation factor $\mathrm{Q}$ and the energy moment $E$ for a family $w(t)$ of windows. (c) Composite desideratum $K$ as a function of alpha for some values of the composition parameter $\gamma$. (d) Lower and upper limits on $K$ as a function of $\gamma$.

is also a window. That is, we have a parametric family of windows, if two different windows are defined.

Let

$$
w_{1}(t)=\cos (\pi t / 2)
$$

and

$$
w_{2}(t)=|\sin (\pi t)| / \pi+(1-|t|) \cos (\pi t),
$$

be two such windows (Papoulis 1977). Some members of the family of windows of (1) are shown in figure 1a. Choice of windows is a difficult practical problem because many criteria exist for the purpose and they do not necessarily tally with each other. Two such criteria are variance compensation factor $Q$ (Prabhu et al 1977) and energy moment $E$ (Papoulis 1977). Both of them should be as small as possible. They are defined as

$$
Q=(1 / 2) \int_{-1}^{1} w^{2}(t) \mathrm{d} t
$$


and

$$
E=\frac{1}{2 \pi} \int_{-\infty}^{\infty} \omega^{2} W^{2}(\omega) \mathrm{d} \omega=\int_{-1}^{1}[w(t)]^{2} \mathrm{~d} t
$$

These parameters for the window $w_{1}(t)$ are

$$
Q_{1}=0.5, \quad E_{1}=\pi^{2} / 4=2.4674 \text {. }
$$

For the window $w_{2}(t)$, they are

$$
Q_{2}=\frac{3}{4 \pi^{2}}+\frac{1}{6}=0.2427, \quad E_{2}=\frac{\pi^{2}}{3}-\frac{1}{2}=2.7899 .
$$

Thus on the criterion $Q$, window $w_{2}(t)$ is better and on the criterion $E$, window $w_{1}(t)$ is better. So, which window should we choose? The choice could be extended to any window $w(t)$ of (1). $Q$ and $E$ are

$$
Q=\frac{\alpha^{2}}{2}+\left(\frac{8}{3 \pi}+\frac{40}{9 \pi^{2}}\right) \alpha(1-\alpha)+\left(\frac{3}{4 \pi^{2}}+\frac{1}{6}\right)(1-\alpha)^{2},
$$

and

$$
E=\frac{\pi^{2} \alpha^{2}}{4}+\frac{32}{9} \alpha(1-\alpha)+\left(\frac{\pi^{2}}{3}-\frac{1}{2}\right)(1-\alpha)^{2}
$$

These parameters are depicted in figure $1 \mathrm{~b}$. The new possibilities opened are obvious. Let the new parameter $K$ be defined as

$$
K=\gamma E_{n}+(1-\gamma) Q_{n}, \quad 0 \leq \gamma \leq 1,
$$

where $E_{n}$ and $Q_{n}$ are versions of $E$ and $Q$ respectively, normalized to lie between [0,1]. $K$ is depicted versus $\alpha$, that is for all the windows of (1), for some discrete values of $\gamma$ in figure 1c. Figure 1d shows the minimum and maximum limits of $K$ versus $\gamma$. Thus, if $\gamma$ deviates from 0 or 1 , that is if the single criterion $Q$ or $E$ is not used, but the convex criterion $K$ is used, its minimum value is no more the best possible, nor is the maximum value the worst possible. If a suitable satisficing or Simonizing value of $\gamma$ is chosen on the basis of figure 1d, the plot of figure 1c can be made for it and the Simonized value of $\alpha$ can be obtained. That determines the Simonized window from (1). These concepts are used in the next section, and with some differences, explained there. In the example, the complexities of multiextremal situation have been deliberately left out.

\section{Simonization}

In the algorithm called linked Simonization, one can start with any sequence and subject it to recursive Hamming scan. At every stage, one goes to a Hamming neighbour which leads to the largest relative improvement $I$, provided it is positive. $I$ is defined as

$$
I=\delta D / D+\gamma \delta M / M
$$

where $\delta D$ and $\delta M$ are the relative improvements in $D$ and $M$ respectively and $\gamma$ is a weighting factor. In the example of windows, the optimization problem was analytically 
tractable. Therefore, it was possible to deal with a convex combination of two criteria after normalization. Here, the optimization is done by a recursive search. Hence, if normalization is used, it has also to be recursive. Instead, relative improvements in the two criteria are combined. We have chosen the weight $\gamma$ to be unity. As the algorithm advances, both $D$ and $M$ may improve simultaneously, or one of them may improve at the cost of the other. In the latter case, the relative improvement in one of them must be more than the weighted relative deterioration in the other. The Hamming scan is terminated when none of these situations is possible. There is another aspect. If one of the objective functions improves at the cost of the other for some steps, the same increment in it will be a smaller percentage for the next step, thus reducing the weightage for it automatically. This is a useful feature, which forbids any objective function from excessively increasing at the cost of the other.

This too is an optimization problem, and is subject to all the trappings of local optimization. Hence, various untrapping techniques (Moharir et al 1997a) such as backtracking, sidetracking, and sidetracking with increased length, have been applied here also.

\section{Results and discussion}

The ternary sequences obtained by Simonization are listed in table 3 . It can be seen that they offer a better solution, simultaneously satisfactory in terms of both the discrimination and the merit factor, though not the best in either of them. The statistical information about the ternary sequences obtained by the linked Simonization algorithm is given in table 4 .

Table 3. Details of some of the ternary sequences obtained by Simonization technique.

Neither discrimination nor merit factor are the best possible, but both of them are satisfactory.

\begin{tabular}{rcc}
\hline Length & Merit factor & Discrimination \\
\hline 24 & 16.6667 & 20.0000 \\
592 & 13.8787 & 32.0833 \\
600 & 13.1630 & 35.1818 \\
599 & 12.5785 & 42.7778 \\
594 & 12.1074 & 38.7000 \\
591 & 11.9341 & 43.5556 \\
623 & 11.0558 & 39.6000 \\
590 & 11.2835 & 39.3000 \\
619 & 10.5876 & 40.0000 \\
590 & 10.2431 & 42.8889 \\
613 & 10.0424 & 39.4000 \\
1564 & 9.9746 & 42.1739 \\
1565 & 9.8654 & 42.1739 \\
601 & 9.7447 & 42.8889 \\
600 & 9.2664 & 42.7778 \\
621 & 9.2574 & 43.8889 \\
603 & 8.5285 & 42.7778 \\
659 & 8.4664 & 40.8182 \\
2775 & 8.0209 & 41.0750 \\
1126 & 7.4422 & 49.3333 \\
1618 & 7.0177 & 48.4000 \\
\hline
\end{tabular}


Tabie 4. Statistics of the ternary sequences obtained by Simonization algorithm.

The number of lengths at which sequences meeting or crossing the prescribed thresholds on the merit factor and the discrimination available is shown.

\begin{tabular}{lcc}
$\begin{array}{l}\text { Merit factor } \\
\text { threshold }\end{array}$ & $\begin{array}{c}\text { Discrimination } \\
\text { threshold }\end{array}$ & $\begin{array}{c}\text { Number of } \\
\text { lengths }\end{array}$ \\
\hline 13 & 20 & 7 \\
13 & 28 & 4 \\
12 & 32 & 16 \\
12 & 36 & 4 \\
11 & 32 & 30 \\
11 & 36 & 12 \\
10 & 32 & 40 \\
10 & 36 & 16 \\
9 & 32 & 70 \\
9 & 36 & 35 \\
8 & 32 & 117 \\
8 & 36 & 54 \\
7 & 40 & 118 \\
7 & 44 & 49 \\
6 & 40 & 240 \\
6 & 44 & 123 \\
6 & 48 & 5 \\
\hline
\end{tabular}

The problem of signal design can be more ambitious than the part of it dealt with here. In radar, the problem is not only of getting peaky autocorrelation, but also that of getting a suitable ambiguity function (Vakman 1968). It should have the smallest width not only along the delay axis, but also along the frequency axis. The fact that the volume under the ambiguity surface is preserved, puts serious constraints on the optimal solution of the problem, and only satisficing or Simonization is possible. Similarly, for spread spectrum applications (Holmes 1982) a set of sequences with two properties is needed

(a) The worst autocorrelation should be as impulse-like as possible, and

(b) the strongest crosscorrelation should be as weak as possible.

For such problems, the notion of Simonization can be extended to that of double or multiple Simonization. One Simonization addresses the fact that there are many qualitative desiderata. They are combined suitably, e.g. through a weighted sum. The second Simonization negotiates the problem that any of the qualitative desiderata can be converted into quantitative measures in many ways. Of these two problems, spread spectrum problem has been solved (Subba Rao 1997) using this notion, and the results are being presented elsewhere.

The authors are grateful to Dr H K Gupta for his support and encouragement. Thanks are also due to the anonymous referees for their useful comments. 


\section{References}

Bernasconi J 1987 Low autocorrelation binary sequences: statistical mechanics and configuration space analysis. J.Phys. 48: 559-567

Ching-Lai H and Masud A S M 1979 Multiple objective decision making -s Methods and applications (Berlin: Springer-Verlag)

Cohon J L 1978 Multiobjective programming and planning (New York: Academic Press)

Cook C E, Bernfeld M 1967 Radar signals (New York: Academic Press)

De Groot C, Wurtz D, Hoffman K H 1992 Low autocorrelation binary sequences: Exact enumeration and optimization by evolutionary strategies. Optimization 23: 369-384

Holmes J K 1982 Coherent spread spectrum techniques (New York: Wiley)

Moharir P S, Subba Rao K 1997 Nonbinary sequences with superior merit factors. J. IETE 43: 49-53

Moharir P S, Maru V M, Singh R 1996 S-K-H algorithm for signal design. Electron. Lett. 32: 1648-1649

Moharir P S, Maru V M, Singh R 1997a Untrapping techniques for radar signal design. Electron. Lett. 33: 631-633

Moharir P S, Maru V M, Singh R 1997b Bi-parental product algorithm for coded waveform design in radar. Sādhanā 22: 589-599

Papoulis A 1977 Signal analysis (Auckland: McGraw-Hill Int.) pp 236-243

Pearl J 1985 Heuristics: intelligent search strategies for computer problem solving (Reading, MA: Addison-Wesley) p 14

Prabhu K M M, Reddy V U, Agrawal J P 1977 Performance comparison of data windows. Electron. Lett. 13: 600-601

Simon H 1956 Rational choice and the structure of the environment. Psychol. Rev. 63: 129

Srinivas N, Deb K 1995 Multiobjective optimization using nondominated sorting in genetic algorithms. Evolutionary Comput. 2: 221-248

Subba Rao K 1997 Optimum signal design for radar and spread spectrum applications. Ph D thesis, Osmania University, Hyderabad, p 249

Vakman D E 1968 Sophisticated signals and uncertainty principle in radar (Berlin: SpringerVerlag) p 224

Weile D S, Michielson E 1996 Integer coded Pareto genetic algorithm design of antenna arrays. Electron. Lett. 19: 1744-1745

Wiener N 1964 God and Golem, Inc. (Cambridge: Mass. Inst. of Technol. Press) pp 50-52

Zeleny M (ed.) 1974 Multiple criterion decision making (Kyoto: Springer-Verlag) 